\title{
Cluster-based Haldane states in spin-1/2 cluster chains
}

\author{
Takanori Sugimoto, ${ }^{1,2,{ }^{*}}$ Katsuhiro Morita, ${ }^{1, \dagger}$ and Takami Tohyama $\odot^{1}$ \\ ${ }^{1}$ Department of Applied Physics, Tokyo University of Science, Katsushika, Tokyo 125-8585, Japan \\ ${ }^{2}$ Advanced Science Research Center, Japan Atomic Energy Agency, Tokai, Ibaraki 319-1195, Japan
}

(Received 23 December 2019; revised manuscript received 7 June 2020; accepted 8 June 2020; published 30 June 2020)

\begin{abstract}
The Haldane state is a typical quantum and topological state of matter, which exhibits an edge state corresponding to symmetry-protected topological order in a one-dimensional integer spin chain. Here we propose a concept to design the Haldane state with one-half spins by making use of a chain composed of one-half spin clusters. If the clusters contain two spins, the ground state of a chain corresponds to the Affleck-Kennedy-LiebTasaki state. We thus extend this knowledge to general clusters consisting of not only even, but odd numbers of one-half spins. In the case of an odd number of spins in the clusters, we present a concrete procedure to construct a field-induced Haldane state, and demonstrate the Haldane state in a five-spin cluster chain. Our concept is useful to understand quantum magnetism in real materials such as Fedotovite, consisting of six-spin clusters aligned in one dimension. Furthermore, the edge state designed by our flexible scheme can be utilized for a processing unit of quantum computation with recent progress on intended synthesis of organic materials, quantum dots, and optical lattices.
\end{abstract}

DOI: 10.1103/PhysRevResearch.2.023420

\section{INTRODUCTION}

The concept of topology has given us clear perspectives on the state of matter annoyed by quantum and/or thermal fluctuations. In condensed matter, the topology provides robust physical properties against disturbance of circumstance. For instance, topologically protected quantum computing has been proposed in a topological superconductor [1-4], and a flat band in topological matters is expected to heighten the critical temperatures of the fractional quantum Hall state [5-7] and surface superconductor [8].

Among many topological matters, one of the simplest models is the Haldane state in a quantum spin chain in which an integer spin is antiferromagnetically coupled to the nearestneighbor spins. This state was first proposed by Haldane in 1983 with a famous conjecture [9]: an antiferromagnetic spin chain of integer spins $(S=1,2,3, \ldots)$ [10] has a finite excitation gap above the ground state. As a characteristic of the $S=1$ Haldane state, Affleck-Kennedy-Lieb-Tasaki (AKLT) have shown a hidden topological order, i.e., string order, in a clear schematic of the rigorous ground state [11-14]. In this theory, an $S=1$ spin is decomposed into two virtual $S=\frac{1}{2}$ spins. As the ground state, the virtual $S=\frac{1}{2}$ spins of neighboring sites are antisymmetrized, and the virtual spins at each site are symmetrized as a real $S=1$ spin. Hence, the

\footnotetext{
*sugimoto.takanori@rs.tus.ac.jp

Present address: Department of Basic Science, University of Tokyo, Meguro, Tokyo 153-8902, Japan.

Published by the American Physical Society under the terms of the Creative Commons Attribution 4.0 International license. Further distribution of this work must maintain attribution to the author(s) and the published article's title, journal citation, and DOI.
}

ground state retains a strong entanglement between neighboring sites, where a finite string order appears. Furthermore, Pollmann et al. have shown a qualitative difference between $S=$ odd and even Haldane chains [15], which is the so-called symmetry-protected topological order.

Recently, Brennen and Miyake proposed the concept of holographic quantum computing using the symmetryprotected topological order in the Haldane state [16]. There are, however, few compounds possessing the $S=$ odd spins in one dimension [16-18]. Here we propose a concept to design the $S=1$ Haldane chain with one-half spin clusters, which will be designable in organic materials [19], quantum dots [20,21], and optical lattices [22,23]. Moreover, our concept enables us to directly observe the entangled quantum state of one-half spins in the Haldane state, which is applicable to the quantum computing at the dawn of designable quantum states based on one-half spins.

\section{MODEL: SPIN CLUSTER CHAINS}

In the present study, we call a model given by the following Hamiltonian a spin cluster chain (SCC):

$$
\mathcal{H}_{0}=\sum_{j=1}^{L} \mathcal{H}_{\text {intra }}^{(j)}+\sum_{j=1}^{L-1} \mathcal{H}_{\text {inter }}^{(j)},
$$

with

$$
\begin{gathered}
\mathcal{H}_{\text {intra }}^{(j)}=\sum_{\left\langle i, i^{\prime}\right\rangle \in B_{\text {intra }}} J_{\left\langle i, i^{\prime}\right\rangle} \boldsymbol{S}_{i, j} \cdot \boldsymbol{S}_{i^{\prime}, j}, \\
\mathcal{H}_{\text {inter }}^{(j)}=\sum_{\left\langle\left\langle i, i^{\prime}\right\rangle\right\rangle \in B_{\text {inter }}} J_{\left\langle\left\langle i, i^{\prime}\right\rangle\right\rangle}^{\prime} \boldsymbol{S}_{i, j} \cdot \boldsymbol{S}_{i^{\prime}, j+1},
\end{gathered}
$$

where $L$ is the number of clusters, and $B_{\text {intra }}=\left\{\left(i, i^{\prime}\right) \mid i, i^{\prime} \in\right.$ $\left.\mathbb{N}, 1 \leqslant i<i^{\prime} \leqslant N\right\}$ and $B_{\text {inter }}=\left\{\left(i, i^{\prime}\right) \mid i, i^{\prime} \in \mathbb{N}, 1 \leqslant i \leqslant\right.$ 


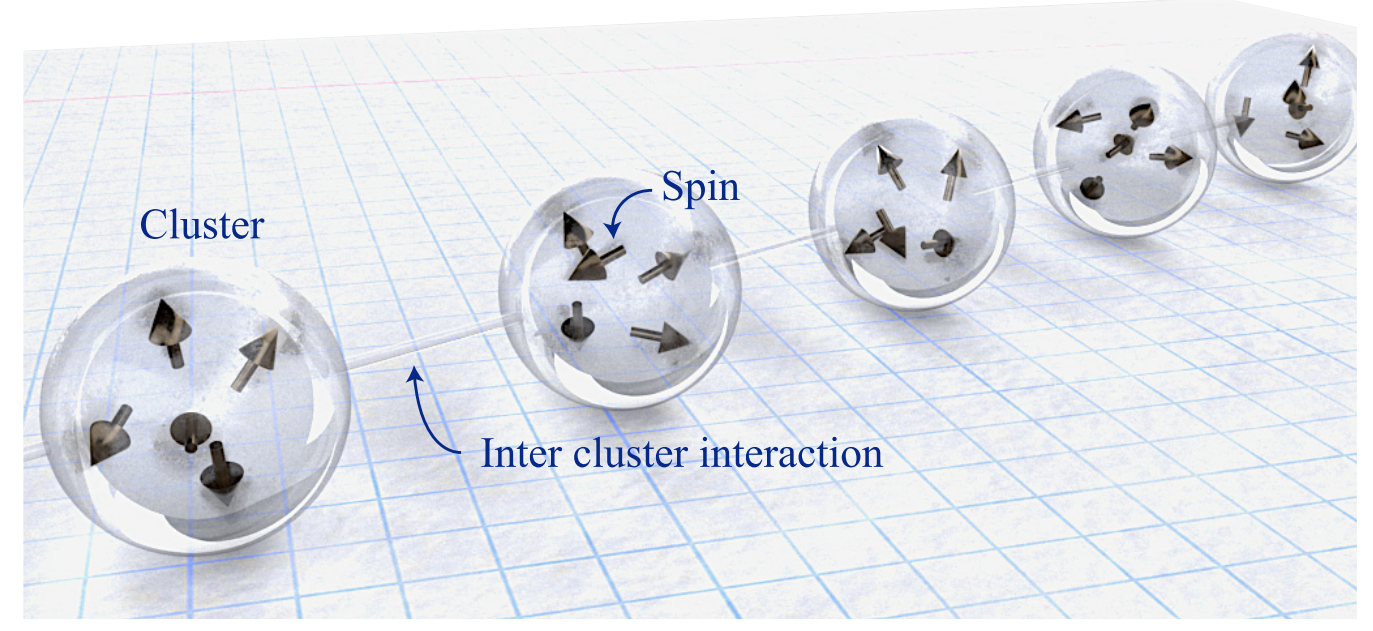

FIG. 1. Schematic ground state of a spin cluster chain (SCC). One-half spins $\left(S=\frac{1}{2}\right)$ are represented by arrows. We assume that spins in a transparent ball (cluster) strongly interact with each other, and that the spins weakly interact with spins in the next balls. The detailed behaviors in three temperature regions are explained in the main text.

$\left.N, 1 \leqslant i^{\prime} \leqslant N\right\}$, with $N$ being the number of spins in a cluster, are a set of intracluster bonds and a set of intercluster bonds, respectively. For simplicity, we consider only the Heisenberg interactions as interactions between one-half spins. As a condition of cluster chain, we assume that the intracluster interaction $J_{\text {intra }}=\sqrt{\sum_{\left\langle i, i^{\prime}\right\rangle \in B_{\text {intra }}}\left(J_{\left\langle i, i^{\prime}\right\rangle}\right)^{2}}$ is much greater than the intercluster interaction $J_{\text {inter }}=\sqrt{\sum_{\left\langle\left\langle i, i^{\prime}\right\rangle\right\rangle \in B_{\text {inter }}}\left(J_{\left\langle\left\langle i, i^{\prime}\right\rangle\right\rangle}^{\prime}\right)^{2}}$, i.e., $J_{\text {intra }} \gg J_{\text {inter }}$. Figure 1 shows the ground state of this model schematically, where a transparent ball including arrows represents a spin cluster, which is connected by a weak intercluster interaction (a transparent bond between balls). Temperature dependence in this system is divided into three stages by two characteristic temperatures $T_{1}<T_{2}$ :

(a) High-temperature region $\left(T>T_{2} \sim J_{\text {intra }}\right)$. Every spin behaves independently such as the Pauli paramagnetism. In Fig. 1, the transparent balls and bonds melt, and every spin (arrow) rotates freely.

(b) Middle-temperature region $\left(T_{1}<T<T_{2}\right)$. The magnetic behavior in this region is understood by an isolated cluster of spins. Here the intercluster interactions are effectively ignored by thermal fluctuation. In Fig. 1, spins included in a ball interact with each other, although the spins do not affect spins belonging to the next balls.

(c) Low-temperature region $\left(T<T_{1} \sim J_{\text {inter }}\right)$. A part of spin degrees of freedom is frozen, so that only the ground/low-energy states in a cluster contribute to manybody interaction due to the intercluster interaction. In Fig. 1, every ball is frozen, and thus spins in a ball do not move independently. However, balls can rotate independently while the intercluster interaction works on them. At zero temperature, the balls definitely stop due to the intercluster interaction as expected in the ground state.

\section{CLUSTER-BASED HALDANE STATES}

Recent experimental study supported by our theoretical analysis has shown that a naturally occurring mineral $\mathrm{Fe}-$ dotovite, $\mathrm{K}_{2} \mathrm{Cu}_{3} \mathrm{O}\left(\mathrm{SO}_{4}\right)_{3}$, is a SCC whose ground state is the Haldane state [24-26]. In this material, six $S=\frac{1}{2}$ spins form an edge-shared tetrahedral cluster, and the clusters stand in line. The ground state in a cluster has been determined by the intracluster interactions, which are estimated by the magnetization curve and the magnetic susceptibility in the middle-temperature region $\left(T_{1}<T<T_{2}\right)$. Since the estimated interaction parameters have indicated that the ground state of Fedotovite is a triplet, the spin degree of freedom corresponding to an $S=1$ spin effectively survives in each cluster at low temperatures. We thus expected the emergence of the Haldane state caused by a weak intercluster interaction. In fact, an inelastic neutron-scattering experiment and magnetization measurement below $T_{1} \sim 4 \mathrm{~K}$ have confirmed the existence of the first excitation gap originating from the intercluster interaction.

What is the condition of the Haldane state in SCCs? To clarify the condition, we start from an $S=\frac{1}{2}$ spin ladder, which exhibits the Haldane state as the ground state, if the rung interaction is ferromagnetic $[27,28]$. Figure 2(a) shows the spin ladder, which corresponds to a two-spin cluster chain whose cluster is a rung, if the cluster condition $\left|J^{\prime}\right| \ll|J|$ is satisfied. When the intracluster interaction is ferromagnetic $J<0$, the ground state is a triplet [Fig. 2(b)], and the low-energy physics is well described by an effective $S=1$ spin. Moreover, the intercluster interaction is rewritten by a Heisenberg interaction between neighboring effective spins. Hence, the effective model at low temperatures is a Heisenberg chain of effective $S=1$ spins, i.e., the $S=1$ Haldane chain. Figure 2(c) shows the schematic ground state of the spin ladder for $J^{\prime}>0$. In the ground state, the spin configuration of a rung is symmetric because of the triplet states, and that of neighboring rungs is antisymmetric due to the antiferromagnetic intercluster interaction. Consequently, this ground state corresponds to the AKLT state, where the real $S=1$ spin is regarded as an effective $S=1$ spin of a rung.

These examples intuitively give us the following conditions to realize the Haldane state in a SCC:

(I) The ground state of a cluster is a triplet, where the total $S^{z}$ of each state changes by 1 . 
(a) Spin ladder

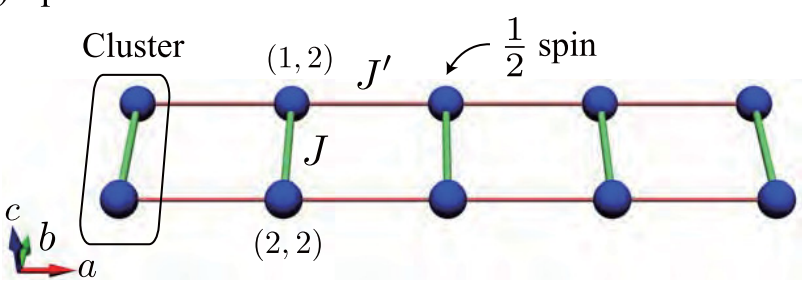

(b) Energy spectrum of a cluster $(J<0)$

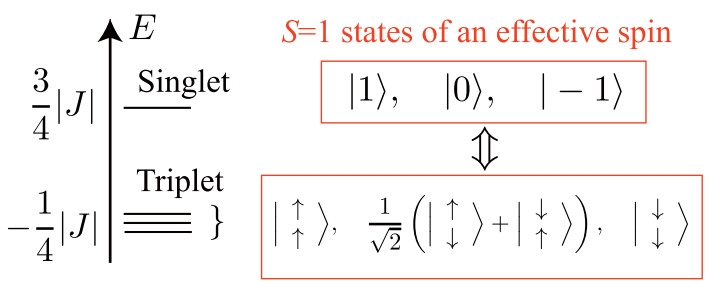

(c) Schematic ground state of a ladder $\left(J^{\prime}>0\right)$

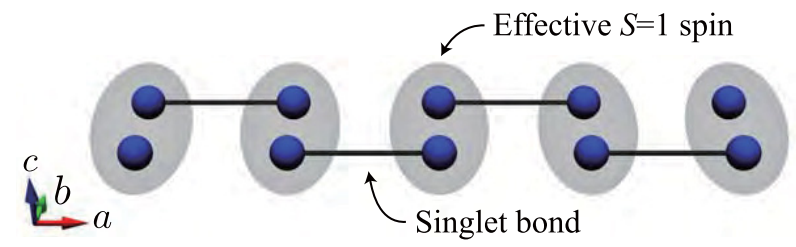

FIG. 2. (a) Spin ladder as a two-spin cluster chain (an $N=2$ $\mathrm{SCC}$ ). The chain is elongated in the $a$ axis. Blue balls and colored segments denote the spin sites and interaction bonds, respectively. The interaction $J\left(J^{\prime}\right)$ corresponds to the intra- (inter)cluster interaction. $(i, j)$ denotes the spin site index, e.g., $(1,2)$ means the first site in the second cluster. (b) Energy spectrum of a cluster (rung) for $J<0$, where the ground state is a triplet. If the intracluster interaction is much smaller than the intercluster interaction $\left|J^{\prime}\right| \ll|J|$, only the triplet states of a cluster contribute to the low-energy physics as an effective $S=1$ spin. (c) Schematic ground state of a SCC for $J^{\prime}>0$. The triplet state of an effective $S=1$ spin is symmetric, and two spins of neighboring rungs are connected with the singlet (antisymmetric) configuration. Thus, this ground state corresponds to the AKLT state.

(II) The intercluster interaction is weak enough as compared with any intracluster interactions.

(III) The effective intercluster interaction between the clusters' ground states is antiferromagnetic and $X X Z$-like $(X Y$, Heisenberg, and weakly Ising types).

We call the ground state satisfying these conditions the cluster-based Haldane state (CBHS), where the string order defined by the effective spins should be observed. Condition I is required to compose an effective $S=1$ spin. Condition II corresponds to an ideal model describing SCCs. Condition III is necessary for creating the Haldane state in an effective model at low temperatures. Note that these correspond to a sufficient condition to exhibit the CBHS.

Condition I (triplet degeneracy) is robust in ideal SCCs where condition II is satisfied. Suppose that the degeneracy is lifted by an additional intracluster term and level separation occurs. Based on condition II, the energy difference between the separated levels should be much larger than the intercluster interactions, leading to the breakdown of CBHS. This is similar to the situation that a single-ion anisotropy $D$ larger than exchange interaction $J_{\mathrm{H}}$ destroys the Haldane state in the $S=1$ antiferromagnetic chain [29], where $D$ and $J_{\mathrm{H}}$ are assumed to correspond to the additional intracluster term and intercluster interactions in our SCC, respectively. Therefore, the triplet should have degeneracy in order to exhibit the CBHSs in ideal SCCs. In addition to the degeneracy, the change of $S^{z}$ by 1 in condition I is necessary for making an effective $S=1$ spin. This change may not be necessary if nonuniform external fields and/or special types of intracluster interactions are added in the SCCs. We leave this interesting problem as a future issue.

In the CBHS, the string and spin correlations are defined by

$$
C_{\mathrm{str}}(r)=\left\langle\tilde{S}_{j}^{z} \exp \left[i \pi \sum_{k=j+1}^{j+r-1} \tilde{S}_{k}^{z}\right] \tilde{S}_{j+r}^{z}\right\rangle, C_{\mathrm{spn}}(r)=\left\langle\tilde{S}_{j}^{z} \tilde{S}_{j+r}^{z}\right\rangle,
$$

where $\tilde{S}_{j}^{z}$ is the $z$ component of the $S=1$ effective spin operator of the $j$ th cluster. For the numerical calculation in this study, we set $j=\frac{L}{2}-\left\lfloor\frac{r}{2}\right\rfloor$, where the floor function $\lfloor x\rfloor$ represents the integer part of $x$. In the following, we illustrate the CBHS with two cases, where the number of spins $N$ in a cluster is either even or odd.

First example: afour-spin cluster chain (an $N=4 S C C$ ). As explained above, the spin cluster in Fedotovite is edge-shared tetrahedra, where six $S=\frac{1}{2}$ spins form a cluster [26]. In the spin ladder model, the cluster contains two $S=\frac{1}{2}$ spins. Both of the cases correspond to the $N=$ even condition, particularly $N=2(\bmod 4)$. Additionally, in Ref. [26], we have examined the CBHS in SCCs where the intracluster interactions have the same symmetry as the cluster of Fedotovite. In the SCCs, the conditions for the CBHS are satisfied when the number of spins in a cluster is $N=2(\bmod 4)$. Is the condition of $N=2(\bmod 4)$ necessary to exhibit the CBHS? The answer is no. As an example of $N=0(\bmod 4)$, we show the CBHS in a four-spin cluster chain given by the following Hamiltonian:

$$
\begin{aligned}
\mathcal{H}_{\text {intra }}^{(j)}= & \frac{J_{\text {intra }}}{2}\left\{\left[\mathrm{Hs}_{(1,2)}^{(j)}-\mathrm{Hs}_{(3,4)}^{(j)}\right]+\cos \theta\left[\mathrm{Hs}_{(1,3)}^{(j)}+\mathrm{Hs}_{(2,4)}^{(j)}\right]\right. \\
& \left.+\sin \theta\left[\mathrm{Hs}_{(1,4)}^{(j)}+\mathrm{Hs}_{(2,3)}^{(j)}\right]\right\}
\end{aligned}
$$

and

$$
\begin{aligned}
\mathcal{H}_{\text {inter }}^{(j)}= & \frac{J_{\text {inter }}}{\sqrt{2}}\left[\cos \phi\left(\mathrm{Hs}_{(2,1)}^{\prime(j)}+\mathrm{Hs}_{(4,3)}^{\prime(j)}\right)\right. \\
& \left.+\sin \phi\left(\mathrm{Hs}_{(2,3)}^{(j)}+\mathrm{Hs}_{(4,1)}^{\prime(j)}\right)\right],
\end{aligned}
$$

where the intra- (inter)cluster Heisenberg interaction $\mathrm{Hs}_{\left(i, i^{\prime}\right)}^{(j)}=$ $\boldsymbol{S}_{i, j} \cdot \boldsymbol{S}_{i^{\prime}, j}\left(\mathrm{Hs}_{\left(i, i^{\prime}\right)}^{\prime(j)}=\boldsymbol{S}_{i, j} \cdot \boldsymbol{S}_{i^{\prime}, j+1}\right)$ and an angle $\theta(\phi)$ control the ratio of intra- (inter)cluster interactions. This model is shown in Fig. 3(a).

Figure 3(b) shows that the ground state of a cluster is triplet (condition I) for $\theta \in\left[\theta_{1}, \theta_{2}\right]$. Since the intracluster Hamiltonian is symmetric with respect to the inversion $\sigma_{j}:\left(\boldsymbol{S}_{1, j}, \boldsymbol{S}_{2, j}, \boldsymbol{S}_{3, j}, \boldsymbol{S}_{4, j}\right) \rightarrow\left(\boldsymbol{S}_{2, j}, \boldsymbol{S}_{1, j}, \boldsymbol{S}_{4, j}, \boldsymbol{S}_{3, j}\right)$, the triplet ground state $\left|t^{\gamma}\right\rangle_{j}(\gamma= \pm, 0)$ satisfies $\sigma_{j}\left|t^{\gamma}\right\rangle_{j}= \pm\left|t^{\gamma}\right\rangle_{j}$, re- 
(a) 4 spin clusters' chain

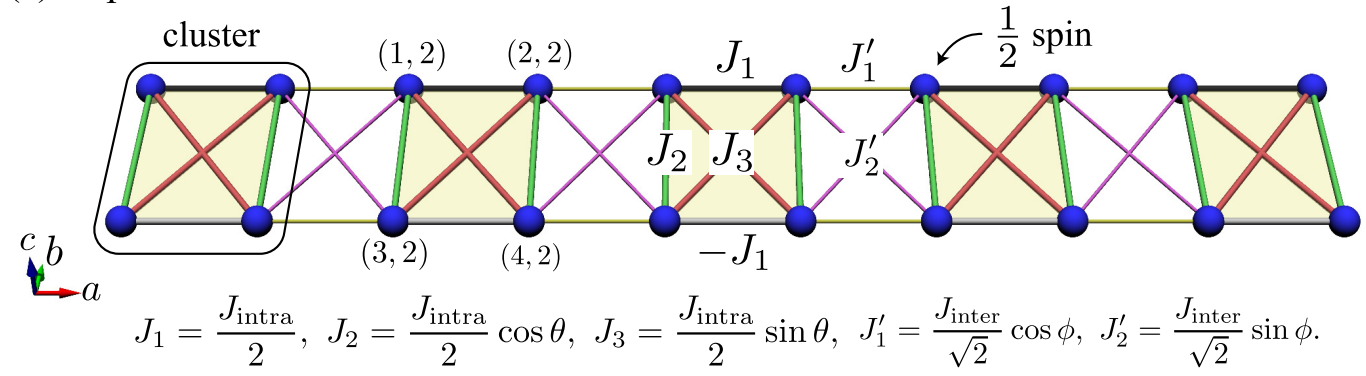

(b) Energy spectrum in a cluster

(c) Correlation function
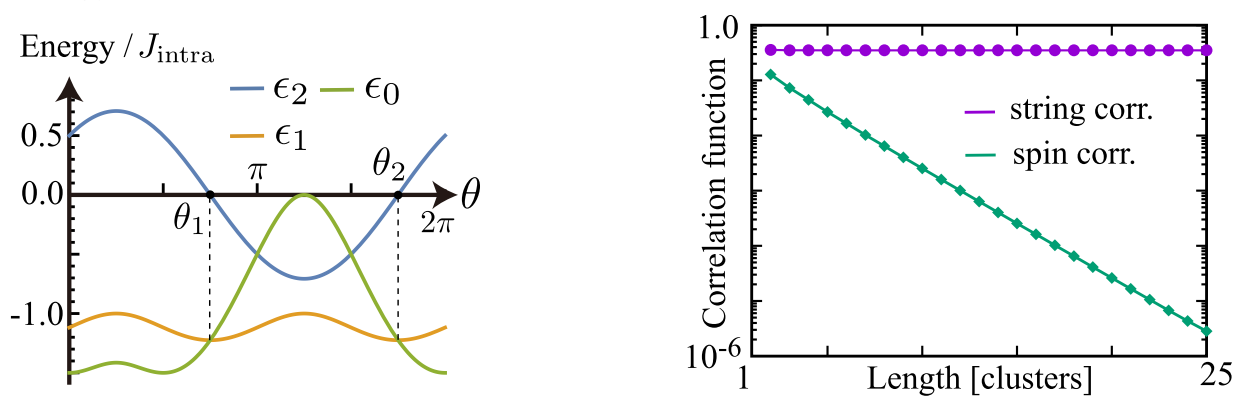

FIG. 3. (a) Four-spin cluster chain (an $N=4$ SCC). Notations are the same as Fig. 2(a). (b) Energy of a cluster as a function of $\theta$ that controls $J_{i}$ with fixed $\phi=\pi / 4$. The lowest-energy level of total spin $(S=0,1$, and 2$)$ is denoted by $\epsilon_{S}$. The energy levels of singlet and triplet states cross at $\theta_{1}=\frac{3}{4} \pi$ and $\theta_{2}=\frac{7}{4} \pi$. (c) Absolute value of string and spin correlation functions for $J_{\text {inter }} / J_{\text {intra }}=0.2, \theta=\frac{5}{4} \pi$, and $\phi=\frac{\pi}{4}$ in a 60 -cluster chain $(L=60)$ with open boundary condition. The correlation functions in (c) are calculated at $M=0$.

sulting in

$$
\begin{aligned}
\left\langle\left. t^{\gamma}\right|_{j}\left(\boldsymbol{S}_{1, j}+\boldsymbol{S}_{3, j}\right) \mid t^{\gamma}\right\rangle_{j} & =\left\langle\left. t^{\gamma}\right|_{j} \sigma_{j}^{2}\left(\boldsymbol{S}_{1, j}+\boldsymbol{S}_{3, j}\right) \sigma_{j}^{2} \mid t^{\gamma}\right\rangle_{j} \\
& =\left\langle\left. t^{\gamma}\right|_{j}\left(\boldsymbol{S}_{2, j}+\boldsymbol{S}_{4, j}\right) \mid t^{\gamma}\right\rangle_{j} .
\end{aligned}
$$

We thus obtain the $S=1$ effective spin operators, $\tilde{S}_{j}^{z}=$ $\left|t^{+}\right\rangle\left\langle t^{+}|-| t^{-}\right\rangle\left\langle t^{-}\right|$and $\tilde{S}_{j}^{ \pm}=\sqrt{2}\left(\left|t^{ \pm}\right\rangle\left\langle t^{0}|+| t^{0}\right\rangle\left\langle t^{\mp}\right|\right)$, by projecting spin operators onto the triplet state through

$$
\begin{aligned}
\mathcal{P}_{j}\left(\boldsymbol{S}_{1, j}+\boldsymbol{S}_{3, j}\right) \mathcal{P}_{j} & =\mathcal{P}_{j}\left(\boldsymbol{S}_{2, j}+\boldsymbol{S}_{4, j}\right) \mathcal{P}_{j} \\
& =\frac{1}{2} \mathcal{P}_{j}\left(\sum_{i} \boldsymbol{S}_{i, j}\right) \mathcal{P}_{j}=\frac{1}{2} \tilde{\boldsymbol{S}}_{j},
\end{aligned}
$$

with the projection operator $\mathcal{P}_{j}=\sum_{\gamma}\left|t^{\gamma}\right\rangle_{j}\left\langle\left. t^{\gamma}\right|_{j}\right.$. Consequently, the intercluster interactions with $\phi=\frac{\pi}{4}$ give the Heisenberg-type interactions between neighboring effective spins (condition III),

$\tilde{\mathcal{H}}=\left(\prod_{j} \mathcal{P}_{j}\right)\left(\sum_{j} \mathcal{H}_{\text {inter }}^{(j)}\right)\left(\prod_{j} \mathcal{P}_{j}\right)=\frac{J_{\text {inter }}}{8} \sum_{j} \tilde{\boldsymbol{S}}_{j} \cdot \tilde{\boldsymbol{S}}_{j+1}$.

Assuming $J_{\text {inter }} \ll J_{\text {intra }}$ (condition II), we should obtain CBHS as the ground state in the four-spin cluster chain. To confirm the existence of the CBHS, we have calculated the correlation functions using the variational matrix product state (VMPS) method [see Fig. 3(c)] [30,31]. In Fig. 3(c), we can find an evidence of the CBHS that the string correlation converges on a constant value, while the spin correlation decreases exponentially with increasing the cluster-cluster distance.
As compared with the first example, the $N=$ odd cases require a little more effort to find the CBHS due to the Kramers' theorem: the energy levels of the system containing an odd number of electrons/spins in the presence of the timereversal symmetry should have evenfold degeneracy. Obviously, this theorem interferes with condition I. To overcome this inconvenience, we introduce the magnetic field into the model Hamiltonian $\mathcal{H}_{0}(1)$, which breaks the time-reversal symmetry and recovers condition I to obtain the CBHS in an $N=$ odd SCC. Since the magnetic field is easily controlled in the laboratory, this procedure is not against our purpose to design the Haldane state with one-half spins for practical use. The magnetic field is introduced by the Zeeman term: $\mathcal{H}=$ $\mathcal{H}_{0}-h^{z} \sum_{i, j} S_{i, j}^{z}$. Since the magnetization $M=\sum_{i, j} S_{i, j}^{z}$ is preserved in the Hamiltonian, the magnetic field just adds the Zeeman energy $-h^{z} M$ to the zero-field energy. Therefore, if the following conditions are satisfied at zero field instead of condition I, a field-induced triplet (FIT) emerges as the ground state at the critical field $h_{c}^{z}$ :

(i) The lowest energy $\epsilon_{S}$ for the total spin $S=S_{0}, S_{0}+$ $1, S_{0}+2$ satisfies the relation that $\Delta=\epsilon_{S_{0}+2}-\epsilon_{S_{0}+1}=$ $\epsilon_{S_{0}+1}-\epsilon_{S_{0}}>0$. Here the critical field is given by $h_{c}^{z}=\Delta$ [see Fig. 4(a)].

(ii) The lowest energy $\epsilon_{S}$ for the total spin $S_{0}$ gives the ground-state energy together with the Zeeman energy at $h^{z}=$ $h_{c}^{z}: \epsilon_{S_{0}}-h_{c}^{z} S_{0} \leqslant \epsilon_{S^{\prime}}-h_{c}^{z} S^{\prime}$ for arbitrary total spin $S^{\prime}$.

How do we find solutions of condition (i) for the $N=$ odd spin cluster? Condition (i) requires three different total spins $S=S_{0}, S_{0}+1, S_{0}+2$, so that $N \geqslant 5$ is required. The number of bonds in an $N \geqslant 5$ cluster is too large to search solutions in the full parameter space, e.g., a five-spin cluster has 10 bonds. In other words, we have to search the solution 
(a) Energy splitting by magnetic field

(b) Decomposition of a cluster
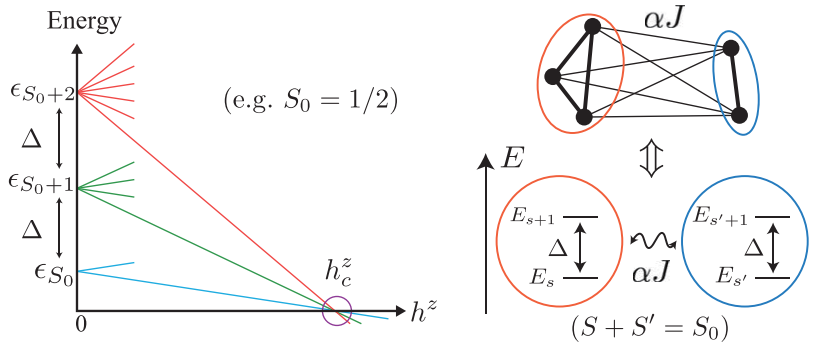

(c) $\delta E$ in parameter space

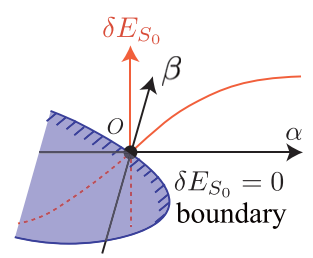

(d) Deformation of a 5 spin cluster

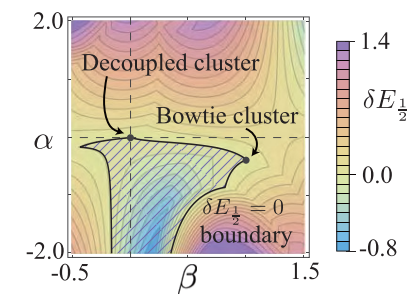

FIG. 4. (a) Schematic of condition (i). If $\epsilon_{S_{0}+2}-\epsilon_{S_{0}+1}=\epsilon_{S_{0}+1}-$ $\epsilon_{S_{0}}=\Delta$ without magnetic fields, a field-induced triplet (FIT) consisting of $\left|S^{z}\right\rangle=\left|\frac{1}{2}\right\rangle,\left|\frac{3}{2}\right\rangle$, and $\left|\frac{5}{2}\right\rangle$ for $S_{0}=1 / 2$ emerges at the critical field $h_{c}^{z}$. (b) Two weakly coupled subsystems denoted by orange and blue circles in a five-spin cluster. The two subsystems are connected by an exchange interaction $\alpha J(J>0$ is the energy unit of the cluster). (c) $\delta E_{S_{0}}=\epsilon_{S_{0}+2}-2 \epsilon_{S_{0}+1}+\epsilon_{S_{0}}$ as a function of $J$ and a deformation parameter $\beta$ independent of $\alpha$. The $\beta=$ 0 case corresponds to (b). The white (purple) region denotes the (positive) negative $\delta E_{S_{0}}$. Since $\delta E_{S_{0}}$ changes its sign at $\alpha=0$ without deformation $(\beta=0)$, we can deform the model parameters along the $\delta E_{S_{0}}=0$ line, on which condition (i) is satisfied. (d) An example of deformation of a five-spin cluster. The point of $(\alpha, \beta)=(0,0)$ corresponds to the trivial case represented in (b). We can reach a simpler model, a bowtie cluster, in Fig. 5(a), as we trace the boundary.

in ten-dimensional parameter space. Instead, we propose the following procedure in the present study. First, we find a trivial solution in a cluster where two subsystems having the same gap $\Delta$ are weakly coupled via an exchange energy $\alpha J$ ( $J>0$ is the energy unit of cluster and $\alpha \ll \Delta / J$ ), as shown in Fig. 4(b). In this model, $\alpha=0$ corresponds to a trivial solution of condition (i), i.e., $\delta E_{S_{0}}=\epsilon_{S_{0}+2}-2 \epsilon_{S_{0}+1}+\epsilon_{S_{0}}=$ 0 , and the sign of $\delta E_{S_{0}}$ changes from negative to positive at $\alpha=0$ with increasing $\alpha$, as explained below. Next, we introduce a parameter $\beta$ independent from $\alpha$ in order to deform the model parameters. The parameter $\beta$ is chosen as one of the model parameters to deform the model into a simpler one: $\beta=0(\beta=1)$ corresponds to the trivial model (a simpler model). For instance, $\beta$ is introduced to decrease the number of interactions or to equalize the strength of several interactions. In the parameter plane of $\alpha$ vs $\beta$, the trivial point is located at $(\alpha, \beta)=(0,0)$. Since $\delta E_{S_{0}}$ is continuous in the parameter space, the boundary between the regions of positive and negative $\delta E_{S_{0}}$ spreads around the trivial point $(\alpha, \beta)=(0,0)$ [see Fig. 4(c)]. Hence, we can trace the boundary satisfying the condition (i), i.e., $\delta E_{S_{0}}=0$, by deforming model parameters from a trivial point to a nontrivial one corresponding to a simpler model in the parameter space.
As mentioned above, we first start by giving a trivial model of a cluster with odd-number spins, where two subsystems of the cluster weakly couple via an exchange energy $\alpha J$. Figure 4(b) shows a five-spin cluster as an example of $N=$ odd clusters, where the cluster is divided into two subsystems of three and two spins. If the two subsystems have the same energy gap $\Delta$ between the lowest levels of the total spin $S$ $\left(S^{\prime}\right)$ and $S+1\left(S^{\prime}+1\right)$, the lowest energy of the total system for the total spin $S_{0}, S_{0}+1$, and $S_{0}+2$ with $S_{0}=S+S^{\prime}$ is given by

$$
\begin{gathered}
\epsilon_{S_{0}}=\epsilon_{S}+\epsilon_{S^{\prime}}+\alpha J S S^{\prime}, \\
\epsilon_{S_{0}+1}= \begin{cases}\epsilon_{S}+\epsilon_{S^{\prime}}+\Delta+\alpha J(S+1) S^{\prime} & (J \geqslant 0) \\
\epsilon_{S}+\epsilon_{S^{\prime}}+\Delta+\alpha J S\left(S^{\prime}+1\right) & (J<0),\end{cases} \\
\epsilon_{S_{0}+2}=\epsilon_{S}+\epsilon_{S^{\prime}}+2 \Delta+\alpha J(S+1)\left(S^{\prime}+1\right) .
\end{gathered}
$$

Since $\delta E_{S_{0}}$ reads

$$
\delta E_{S_{0}}= \begin{cases}\alpha J\left(S-S^{\prime}+1\right) & (\alpha \geqslant 0) \\ \alpha J\left(S^{\prime}-S+1\right) & (\alpha<0),\end{cases}
$$

condition (i) given by $\delta E_{S_{0}}=0$ is satisfied when $\alpha=0$. We note that $\delta E_{S_{0}}$ changes its sign at $\alpha=0$ if $S=S^{\prime}+\frac{1}{2}$. This sign change is useful to find a simpler model in the parameter space of $\alpha$ and $\beta$.

Figure 4(c) shows a schematic behavior of $\delta E_{S_{0}}$ in the $\alpha$ vs $\beta$ space. The white (purple) region in the $\alpha-\beta$ plane denotes the (positive) negative $\delta E_{S_{0}}$, so that the boundary of the two regions denoted by the solid black line stretches around the trivial point $(\alpha, \beta)=(0,0)$. Consequently, we can deform the model parameters by tracing the $\delta E_{S_{0}}=0$ line from the trivial point [see Fig. 4(c)]. Based on this strategy, we have tried several deformations of parameters by changing $\beta$ on the boundary to obtain a simpler model. As an example, we can find the following deformed Hamiltonian of a five-spin cluster with an additional angle $\lambda$ controlling interactions in each subsystem:

$$
\mathcal{H}(\lambda, \alpha, \beta)^{(\mathrm{clst})}=J\left[\mathcal{H}_{1}^{(\mathrm{clst})}+\mathcal{H}_{2}^{(\mathrm{clst})}+\mathcal{H}_{3}^{(\mathrm{clst})}\right],
$$

with

$$
\begin{gathered}
\mathcal{H}_{1}^{\text {(clst) }}=\cos \lambda \boldsymbol{S}_{1} \cdot \boldsymbol{S}_{2}+\frac{\sin \lambda}{\sqrt{2}}\left(\boldsymbol{S}_{1}+\boldsymbol{S}_{2}\right) \cdot \boldsymbol{S}_{3}, \\
\mathcal{H}_{2}^{(\mathrm{clst})}=\xi_{\beta} \boldsymbol{S}_{4} \cdot \boldsymbol{S}_{5}, \\
\mathcal{H}_{3}^{(\mathrm{clst})}=\alpha\left[\frac{\sin \zeta_{\beta}}{2}\left(\boldsymbol{S}_{1}+\boldsymbol{S}_{2}\right) \cdot\left(\boldsymbol{S}_{4}+\boldsymbol{S}_{5}\right)\right. \\
\left.+\frac{\cos \zeta_{\beta}}{\sqrt{2}} \boldsymbol{S}_{3} \cdot\left(\boldsymbol{S}_{4}+\boldsymbol{S}_{5}\right)\right],
\end{gathered}
$$

where the deformation parameter $\beta$ determines two coefficients,

$\xi_{\beta}=\cos \lambda+(1-\beta) \frac{\sin \lambda}{2 \sqrt{2}}, \quad \zeta_{\beta}=(1-\beta) \tan ^{-1}(\sqrt{2})+\beta \pi$.

In this model, $\beta=0$ gives the situation of Fig. 4(b), i.e., $\mathcal{H}_{1}^{\text {(clst) }}$ for one subsystem $\left\{\boldsymbol{S}_{1}, \boldsymbol{S}_{2}, \boldsymbol{S}_{3}\right\}$ and $\mathcal{H}_{2}^{\text {(clst) }}$ for the other $\left\{\boldsymbol{S}_{4}, \boldsymbol{S}_{5}\right\}$, which are connected by the weak coupling $\alpha$, have the same energy gap. On the other hand, $\beta=1$ corresponds 
(a) 5 spin clusters' chain

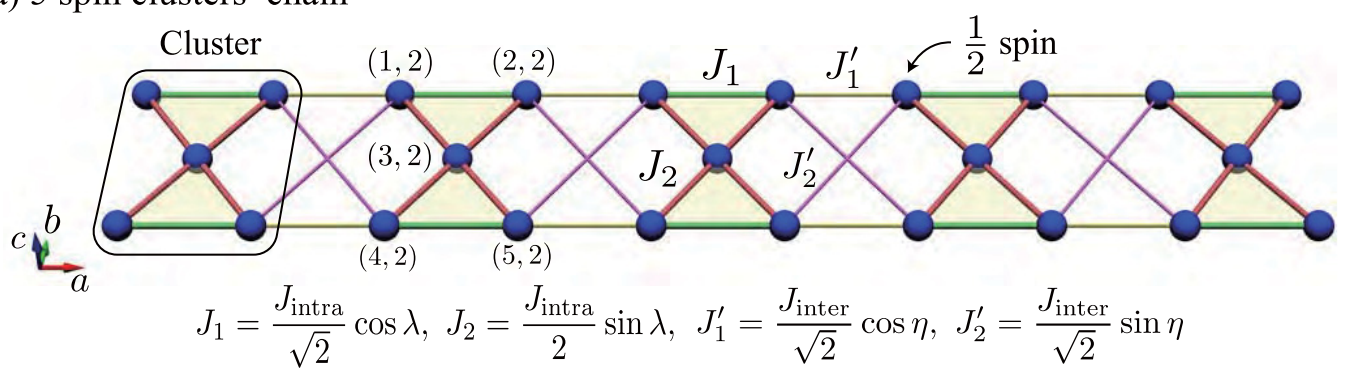

(b) Energy spectrum in a cluster
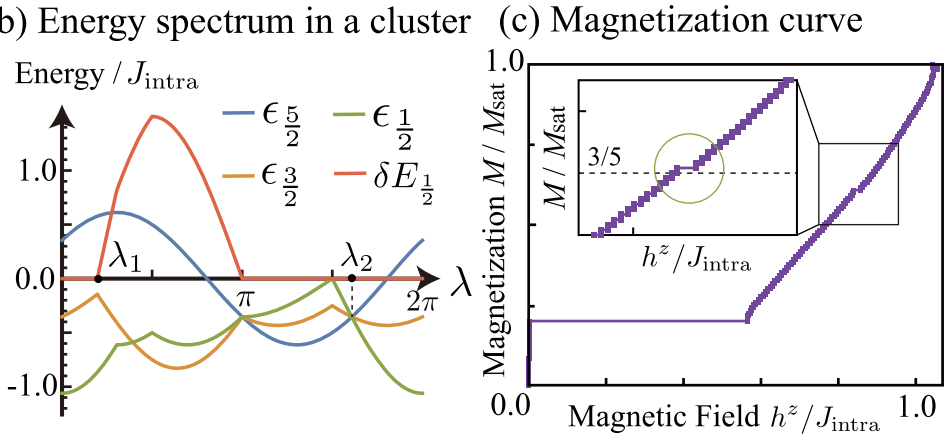

(d) Correlation function

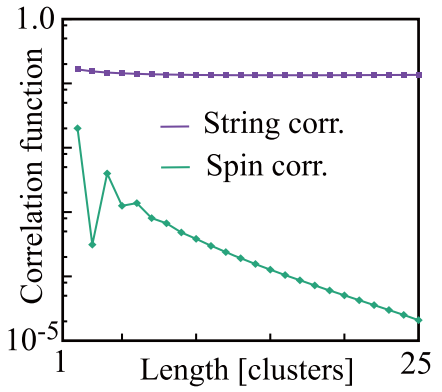

FIG. 5. (a) Five-spin cluster chain (an $N=5$ SCC). Notations are the same as Fig. 2(a). (b) Energy of a cluster as a function of $\lambda$ that controls $J_{i}$ with fixed $\eta=\pi / 4$. Here, $\lambda_{1}=\frac{\pi}{4}-\frac{1}{2} \sin ^{-1}\left(\frac{1}{3}\right)$ and $\lambda_{2}=\frac{3}{2} \pi+\sin ^{-1}\left(\frac{1}{3}\right)$. (c) Magnetization curve normalized by $M_{\text {sat }}$ and (d) absolute value for string and spin correlation functions for $J_{\text {inter }} / J_{\text {intra }}=0.3, \lambda=\frac{\pi}{8}$, and $\eta=\frac{\pi}{4}$ in a 48-cluster chain $(L=48)$ with open boundary condition. The inset of (c) is an enlarged view around $M=\frac{3}{5} M_{\text {sat }}$, where we find the magnetization plateau at $M=\frac{3}{5} M_{\text {sat }}+1$ denoted by a circle. The correlation functions in (d) are calculated at $M=\frac{3}{5} M_{\mathrm{sat}}+1$.

to a simpler model where the coupling constant $\sin \zeta_{\beta}=0$, $\cos \zeta_{\beta}=-1$, and $\xi_{\beta}=\cos \lambda$. Figure 4(d) shows the color map of $\delta E_{\frac{1}{2}}$ in the $\alpha-\beta$ plane with a fixed $\lambda=\frac{\pi}{8}$. We can find that the $\delta E_{S_{0}}=0$ boundary connects between $(\alpha, \beta)=(0,0)$ and $(\alpha, \beta)=\left(\alpha_{c}, 1\right)$ with $\alpha_{c}=-\sin \lambda$. We thus obtain the following simpler model, which we call a bowtie cluster, as a solution of condition (i):

$$
\begin{aligned}
\mathcal{H}\left(\lambda, \alpha_{c}, 1\right)^{(\mathrm{clst})}= & \cos \lambda\left(\boldsymbol{S}_{1} \cdot \boldsymbol{S}_{2}+\boldsymbol{S}_{4} \cdot \boldsymbol{S}_{5}\right) \\
& +\frac{\sin \lambda}{\sqrt{2}} \boldsymbol{S}_{3} \cdot\left(\boldsymbol{S}_{1}+\boldsymbol{S}_{2}+\boldsymbol{S}_{4}+\boldsymbol{S}_{5}\right) .
\end{aligned}
$$

Second example: a five-spin cluster chain (an $N=5$ SCC). As discussed above, we have found that the five-spin cluster described by $\mathcal{H}\left(\lambda, \alpha_{c}, 1\right)^{\text {(clst) }}$ (17) satisfies condition (i) for $\lambda=\frac{\pi}{8}$. To confirm the CBHS in the SCC constructed by $\mathcal{H}\left(\lambda, \alpha_{c}, 1\right)^{(\mathrm{clst})}$, we consider the following SCC model [see Fig. 5(a)]:

$$
\begin{aligned}
\mathcal{H}_{\text {intra }}^{(j)}= & \frac{J_{\text {intra }}}{\sqrt{2}}\left\{\cos \lambda\left[\mathrm{Hs}_{(1,2)}^{(j)}+\mathrm{Hs}_{(4,5)}^{(j)}\right]\right. \\
& \left.+\frac{\sin \lambda}{\sqrt{2}}\left[\mathrm{Hs}_{(1,3)}^{(j)}+\mathrm{Hs}_{(2,3)}^{(j)}+\mathrm{Hs}_{(3,4)}^{(j)}+\mathrm{Hs}_{(3,5)}^{(j)}\right]\right\}
\end{aligned}
$$

and

$$
\begin{aligned}
\mathcal{H}_{\text {inter }}^{(j)}= & \frac{J_{\text {inter }}}{2}\left[\cos \eta\left(\mathrm{Hs}_{(2,1)}^{\prime(j, j+1)}+\mathrm{Hs}_{(5,4)}^{\prime(j, j+1)}\right)\right. \\
& \left.+\sin \eta\left(\mathrm{Hs}^{\prime}{ }_{(2,4)}^{(j, j+1)}+\mathrm{Hs}_{(5,1)}^{\prime(j, j+1)}\right)\right] .
\end{aligned}
$$

Figure 5(b) shows the lowest energy for the total spin $S=$ $\frac{1}{2}, \frac{3}{2}, \frac{5}{2}$ and $\delta E_{\frac{1}{2}}$. Interestingly, we can see that condition (i) for $S_{0}=\frac{1}{2}$ is satisfied in $\lambda \in\left[0, \lambda_{1}\right]$ or $\left[\lambda_{2}, 2 \pi\right]$ [32]. When $\lambda \in\left[\lambda_{2}, 2 \pi\right], \epsilon_{\frac{1}{2}}$ gives the lowest energy, which is the same as the case for $\lambda \in\left[0, \lambda_{1}\right]$.

We therefore expect the FIT as the ground state of a cluster at the critical magnetic field [condition (ii)], where the effective spin operator with $S=1$ is given by $\tilde{S}_{j}^{z}=\left|\frac{5}{2}\right\rangle_{j}\left\langle\left.\frac{5}{2}\right|_{j}-\right.$ $\left|\frac{1}{2}\right\rangle_{j}\left\langle\left.\frac{1}{2}\right|_{j}\right.$. Since the projection operator into the FIT space is given by $\mathcal{P}_{j}=\sum_{m=\frac{1}{2}, \frac{3}{2}, \frac{5}{2}}|m\rangle_{j}\left\langle\left. m\right|_{j}\right.$, we obtain the projected magnetization of a cluster $\mathcal{P}_{j} M_{j} \mathcal{P}_{j}=\sum_{i} \mathcal{P}_{j} S_{i, j}^{z} \mathcal{P}_{j}=$ $\sum_{m=\frac{1}{2}, \frac{3}{2}, \frac{5}{2}} m|m\rangle_{j}\left\langle\left. m\right|_{j}\right.$, so that the $z$ component of the effective spin corresponds to the magnetization of a cluster decreased by $\frac{3}{2}, \tilde{S}_{j}^{z}=\mathcal{P}_{j}\left(M_{j}-\frac{3}{2}\right) \mathcal{P}_{j}$. This correspondence means that the effective $S=1$ spin states are given by $|\tilde{1}\rangle_{j} \cong\left|\frac{5}{2}\right\rangle_{j},|\tilde{0}\rangle_{j} \cong$ $\left|\frac{3}{2}\right\rangle_{j}$, and $|\tilde{-1}\rangle_{j} \cong\left|\frac{1}{2}\right\rangle_{j}$. Thus, the Haldane gap can emerge at $\left\langle M_{j}\right\rangle=\frac{3}{2}$, i.e., $M=\sum_{j}\left\langle M_{j}\right\rangle=\frac{3}{5} M_{\text {sat }}$, where $M_{\text {sat }}=\frac{5}{2} L$ is the saturated magnetization. Note that the CBHS in an $N=$ odd SCC is induced by the magnetic field, so that we call it the field-induced Haldane state [33]. To confirm this gap, we have calculated the magnetization curve with a weak intercluster interaction using the VMPS method [see Fig. 5(c)]. In Fig. 5(c), the small magnetization plateau is observed at $M=\frac{3}{5} M_{\text {sat }}+1$ because the Haldane state has free spins at the edges of the chain. Moreover, we confirm the existence of string order based on the effective spins in Fig. 5(d), while the spin correlation decreases exponentially.

\section{DISCUSSION}

In this paper, we restrict conditions of the SCCs to the Heisenberg interactions, for simplicity, and apply the uniform magnetic field to break the time-reversal symmetry. 
These assumptions are introduced as we take experimental feasibility into account. On the other hand, if we consider nonuniform external fields and/or special types of intracluster interactions such as Ising anisotropy and ring exchange, the CBHS in a three-spin cluster chain (an $N=3 \mathrm{SCC}$ ) is available. For instance, there is a possibility of the CBHS as an $M=\frac{1}{3} M_{\text {sat }}+1$ magnetization plateau in the SCC, which has a uniform ferromagnetic exchange interaction with small $X Y$-like anisotropy in each cluster, and has an antiferromagnetic exchange interaction between two spins in neighboring clusters, because in a certain magnetic field, we can find a pseudodegeneracy of $\left|S^{z}\right\rangle=\left|-\frac{1}{2}\right\rangle,\left|\frac{1}{2}\right\rangle$, and $\left|\frac{3}{2}\right\rangle$ states in each cluster as low-lying states instead of the FIT. In this model, the configuration of CBHS is expected to be similar to the field-induced Haldane state in the $S=1 / 2$ ferroferro-antiferromagnetic (FFAF) chain [33]. We leave other interesting problems in SCCs including exotic many-body interactions as a future issue.

\section{CONCLUSION}

In summary, we have studied the CBHS emerging in SCCs at low temperatures, motivated by the discovery of the CBHS in Fedotovite. The CBHSs have been found in SCCs of onehalf spins which contain not only an even number of spins, but also an odd number of spins. For the cluster with the evennumber spins, the three conditions I-III are useful for finding the CBHS in SCCs. For the odd-number case, condition I is replaced by the two conditions (i) and (ii) and magnetic fields have to be applied. To find a simpler model that exhibits the Haldane state for the odd-number case, we have proposed a procedure of deforming parameters starting from a trivial point where condition (i) is satisfied in the original parameter space. Using this procedure, we have found a simpler model exhibiting the CBHS evidenced by the magnetization plateau and the string order, i.e., a field-induced Haldane state. Since the Haldane state is proposed to be a possible holographic quantum computer, our concept of designable spin systems exhibiting the CBHS is useful for its application.

\section{ACKNOWLEDGMENTS}

We would like to thank M. Fujihala and S. Mitsuda for valuable discussions. This work was partly supported by a Grant-in-Aid for Young Scientists (B) (Grant No. 16K17753), Grant-in-Aid for Scientific Research (C) (Grant No. 20K03840), the CDMSI project on a post-K computer, and the interuniversity cooperative research program of IMR, Tohoku University. Numerical computation in this work was carried out on the supercomputers at JAEA and the Supercomputer Center at the Institute for Solid State Physics, University of Tokyo.
[1] N. Read and D. Green, Phys. Rev. B 61, 10267 (2000).

[2] A. Y. Kitaev, Phys. Usp. 44, 131 (2001).

[3] D. A. Ivanov, Phys. Rev. Lett. 86, 268 (2001).

[4] L. Mazza, M. Rizzi, M. D. Lukin, and J. I. Cirac, Phys. Rev. B 88, 205142 (2013).

[5] E. Tang, J.-W. Mei, and X.-G. Wen, Phys. Rev. Lett. 106, 236802 (2011).

[6] K. Sun, Z. Gu, H. Katsura, and S. Das Sarma, Phys. Rev. Lett. 106, 236803 (2011).

[7] T. Neupert, L. Santos, C. Chamon, and C. Mudry, Phys. Rev. Lett. 106, 236804 (2011).

[8] N. B. Kopnin, T. T. Heikkilä, and G. E. Volovik, Phys. Rev. B 83, 220503(R) (2011).

[9] F. D. M. Haldane, Phys. Lett. A 93, 464 (1983); Phys. Rev. Lett. 50, 1153 (1983).

[10] In this article, we use the natural unit $\hbar=1$.

[11] I. Affleck, T. Kennedy, E. Lieb, and H. Tasaki, Commun. Math. Phys. 115, 477 (1988).

[12] M. den Nijs and K. Rommelse, Phys. Rev. B 40, 4709 (1989).

[13] H. Tasaki, Phys. Rev. Lett. 66, 798 (1991).

[14] T. Kennedy and H. Tasaki, Phys. Rev. B 45, 304 (1992).

[15] F. Pollmann, E. Berg, A. M. Turner, and M. Oshikawa, Phys. Rev. B 85, 075125 (2012).

[16] G. K. Brennen and A. Miyake, Phys. Rev. Lett. 101, 010502 (2008); A. Miyake, ibid. 105, 040501 (2010).

[17] D. Gross and J. Eisert, Phys. Rev. Lett. 98, 220503 (2007).

[18] D. V. Else, I. Schwarz, S. D. Bartlett, and A. C. Doherty, Phys. Rev. Lett. 108, 240505 (2012).
[19] For a review, see T. Ishiguro, K. Yamaji, and G. Saito, Organic Superconductors (Springer-Verlag, Berlin, 1998).

[20] B. E. Kane, Nature (London) 393, 133 (1998).

[21] D. Loss and D. P. DiVincenzo, Phys. Rev. A 57, 120 (1998).

[22] J. I. Cirac and P. Zoller, Phys. Rev. Lett. 74, 4091 (1995).

[23] M. Greiner, O. Mandel, T. Esslinger, T. W. Hänsch, and I. Bloch, Nature (London) 415, 39 (2002).

[24] L. P. Vergasova, S. K. Filatov, Y. K. Serafimova, and G. L. Starova, Doklady Acad. Nauk. SSSR 299, 961 (1988).

[25] G. L. Starova, S. K. Filatov, V. S. Fundamensky, and L. P. Vergasova, Mineral. Mag. 55, 613 (1991).

[26] M. Fujihala, T. Sugimoto, T. Tohyama, S. Mitsuda, R. A. Mole, D. H. Yu, S. Yano, Y. Inagaki, H. Morodomi, T. Kawae, H. Sagayama, R. Kumai, Y. Murakami, K. Tomiyasu, A. Matsuo, and K. Kindo, Phys. Rev. Lett. 120, 077201 (2018).

[27] T. Masuda, A. Zheludev, H. Manaka, L.-P. Regnault, J.-H Chung, and Y. Qiu, Phys. Rev. Lett. 96, 047210 (2006).

[28] T. Vekua and A. Honecker, Phys. Rev. B 73, 214427 (2006).

[29] W. Chen, K. Hida, and B. C. Sanctuary, Phys. Rev. B 67, 104401 (2003).

[30] U. Schollwöck, Annal. Phys. 326, 96 (2011).

[31] In VMPS calculations, we set the number of kept states $m=$ 300 , as we confirm that the error of truncated states is less than $10^{-8}$

[32] The region $\left[\pi, \lambda_{2}\right]$ is not suitable for condition (i) because the gap $\Delta$ is negative, i.e., $\epsilon_{\frac{5}{2}}\left(\epsilon_{\frac{3}{2}}\right)$ is lower than $\epsilon_{\frac{3}{2}}\left(\epsilon_{\frac{1}{2}}\right)$.

[33] As another example of a field-induced Haldane state in the $S=1 / 2$ FFAF chain, which is not a SCC, see S. Takayoshi, K. Totsuka, and A. Tanaka, Phys. Rev. B 91, 155136 (2015). 